\title{
A differential equation model, finding the drowning point when search for crashed planes
}

\author{
Nan Wang \\ School of North China Electric Power University (Baoding), Baoding 071001, China; \\ Email:349968761@qq.com
}

Keywords: differential equation model , Runge-Kutta method

\begin{abstract}
To solve searching problem, we will establish a model to describe the trajectory of the falling and determine the drowning point. With the application of kinematics knowledge, we obtain relationship between time and displacement in the both horizontal and vertical directions. We use linear fitting to draw the air density-altitude curve considering the changes of air density, so that we can establish a differential equation model and use Runge-Kutta method to get the numerical solution, then we can draw the falling curve in the two-dimensional plane.
\end{abstract}

\section{Introduction}

we analysis the movement of the plane after it loses it power, basing on the flight dynamics. And seek the image of airplane's horizontal and vertical displacement with regard to the fall time using the obtained curve by fitting the air density - evaluation. And then establish differential equation model, so we can get the plane crashed trajectory and the most possible falling points of the airplane.

\section{Model Establishment}

We consider the vertical projection of the point where the airplane loses its power on the sea level as the coordinate origin. We put the horizontal axis in north-east, and the vertical axis in the vertical direction. That's the Cartesian coordinate system below:

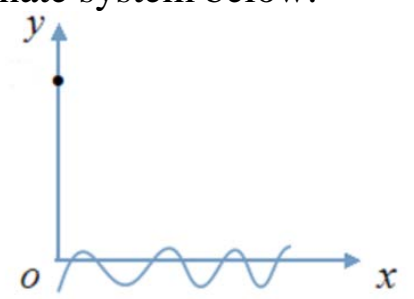

To describe the trajectory after the plane loses its power, firstly we need to do stress analysis of the airplane.

Considering the air-stream and according to the analysis above, the airplane suffer lift force F, stress drag f and gravity $\mathrm{G}$. The force diagram is shown below:

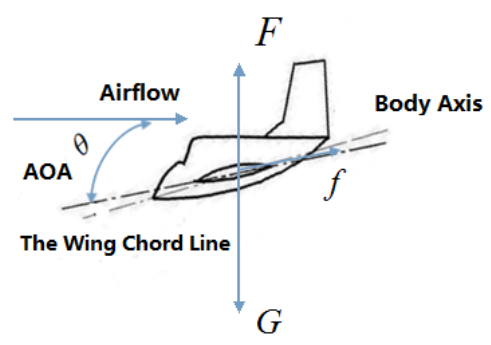

By Newton's second law, is

$$
\left\{\begin{array}{l}
M a_{x}=-f \cos \theta \\
M a_{y}=F+f \sin \theta-M g
\end{array}\right.
$$

By literature [1], combined with the analysis of 5.1.1, stress drag is the main resistance: 
Additionally, by literature [2], the lift force that the airplane suffer is:

$$
f=\frac{1}{16} S_{1} C_{w} v^{2}
$$

Then we can get the differential equations:

$$
F=\frac{1}{2} \rho S_{2} C_{F} v^{2}
$$

It means that we get the differential equation model of the falling locus.

$$
\left\{\begin{array}{l}
\frac{d^{2} x}{d t^{2}}=-\frac{S_{1} C_{W}}{16 M}\left[\left(\frac{d x}{d t}\right)^{2}+\left(\frac{d y}{d t}\right)^{2}\right] \cos \theta \\
\frac{d^{2} y}{d t^{2}}=-g+\frac{\left(S_{1} C_{W} \sin \theta+8 \rho S_{2} C_{F}\right)}{16 M}\left[\left(\frac{d x}{d t}\right)^{2}+\left(\frac{d y}{d t}\right)^{2}\right]
\end{array}\right.
$$

Combined with the initial conditions:

$$
x(0)=0, \quad y(0)=0
$$

The answer can be solved.

\section{Model Solution}

Firstly we need to obtain the value of the relevant parameters in equation (4).

According to the assumption, $S_{1} 、 S_{2} 、 C_{w}$ don't change. In this solution, we set MH370 (Boeing 777-200) as an example. Given that it's cruising speed is $v_{h}$, and its unit is Mach.

Relevant parameters as follows

\begin{tabular}{|c|c|c|c|c|}
\hline$M$ & $S_{1}$ & $S_{2}$ & $v_{h}$ & $C_{w}$ \\
\hline 297550 & 110 & 845 & 0.85 & 0.08 \\
\hline
\end{tabular}

We take the data in the table into this model and we still need to obtain the lift coefficient $C_{F}$ and air density $\rho$.Here we solve them respectively.

\subsection{The Solution of the lift coefficient $C_{F}$}

By literature [2], when the airplane is in the air, the forces that it suffers in the vertical direction are balanced. In the vertical direction, it has lift upright and gravity downward. By Newton's first law, there is

$$
F=M g
$$

Put equations [3] [6] together, we can get

$$
C_{F}=0.2010
$$

\subsection{Fitting the air density-altitude curve}

According to the Standard Atmosphere Table, we can get part of the data of the relationship between air density and altitude during the falling. 


\begin{tabular}{|c|c|}
\hline Altitude $(m)$ & Air density $\left(\mathrm{kg} / \mathrm{m}^{3}\right)$ \\
\hline 0 & 1.225 \\
\hline 1000 & 1.111 \\
\hline 2000 & 1.007 \\
\hline 3000 & 0.9093 \\
\hline 4000 & 0.8194 \\
\hline 5000 & 0.7364 \\
\hline 6000 & 0.6601 \\
\hline 7000 & 0.5900 \\
\hline 8000 & 0.5258 \\
\hline 9000 & 0.4671 \\
\hline 10000 & 0.4135 \\
\hline
\end{tabular}

Does first-order linear fitting using the data above, we can draw air density-altitude curve, like this:

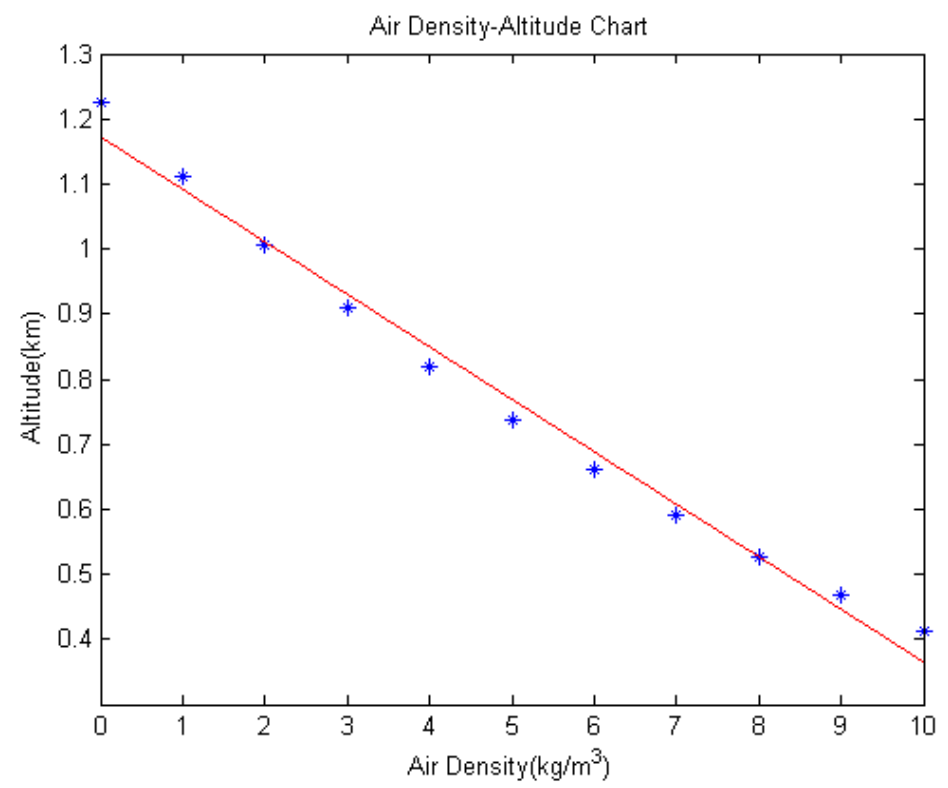

3.3 Solution to the falling locus

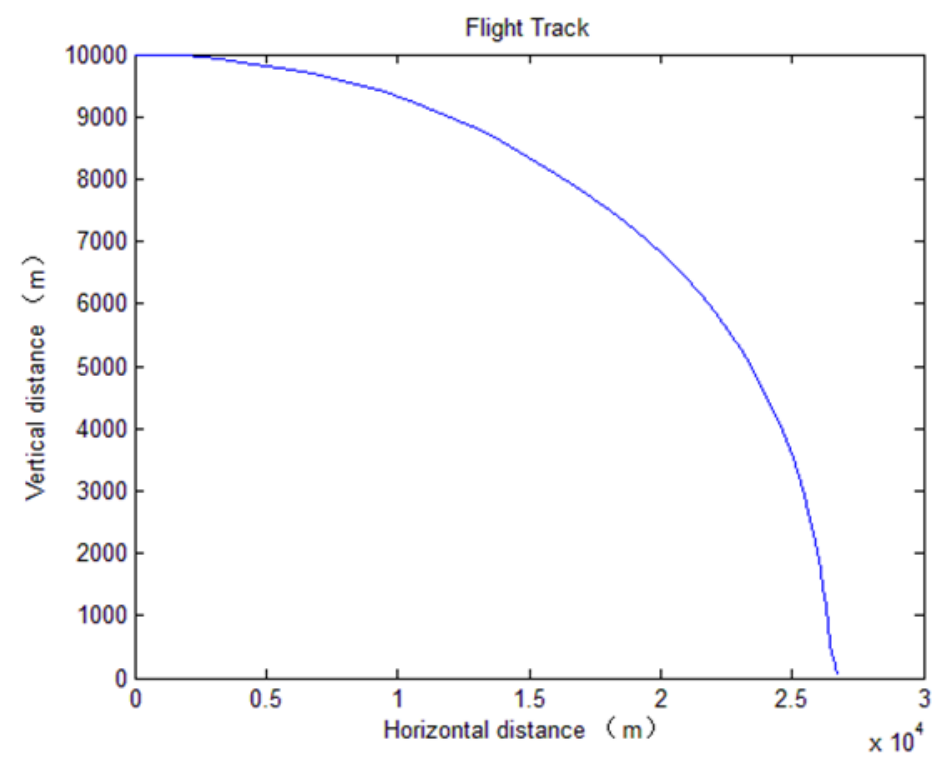


Finding that there is no analytical solution to the differential equation (4) in 5.1.2, we use Runge-Kutta method to get its Numerical solution with the help of MATLAB. Then we draw the falling curve.

We obtain that the distance between the falling location and the drowning point is 26687 meters. The information shows that the lose-contact point is $6.92{ }^{\circ} \mathrm{N}, 103.57^{\circ} \mathrm{E}$. It means that the maximum-possible-drowning point is $7.01^{\circ} \mathrm{N}, 103.79^{\circ} \mathrm{E}$.

\section{Summary}

In practice, parameters related to airplane need to be changed due to different airplane types. The former example is Boeing 777-200(Malaysia Airlines MH370). Now we will replace it with Boeing 747-100 and do comparative analysis.

Parameters of Boeing 747-100 of the parameters are as follow:

\begin{tabular}{|c|c|c|c|}
\hline$M$ & $l$ & $w$ & $h$ \\
\hline 333390 & 35.3 & 29.8 & 9.78 \\
\hline
\end{tabular}

Comparison Chart of Model 1 is below(Picture left is Boeing 747-100,right is Boeing 777-200)
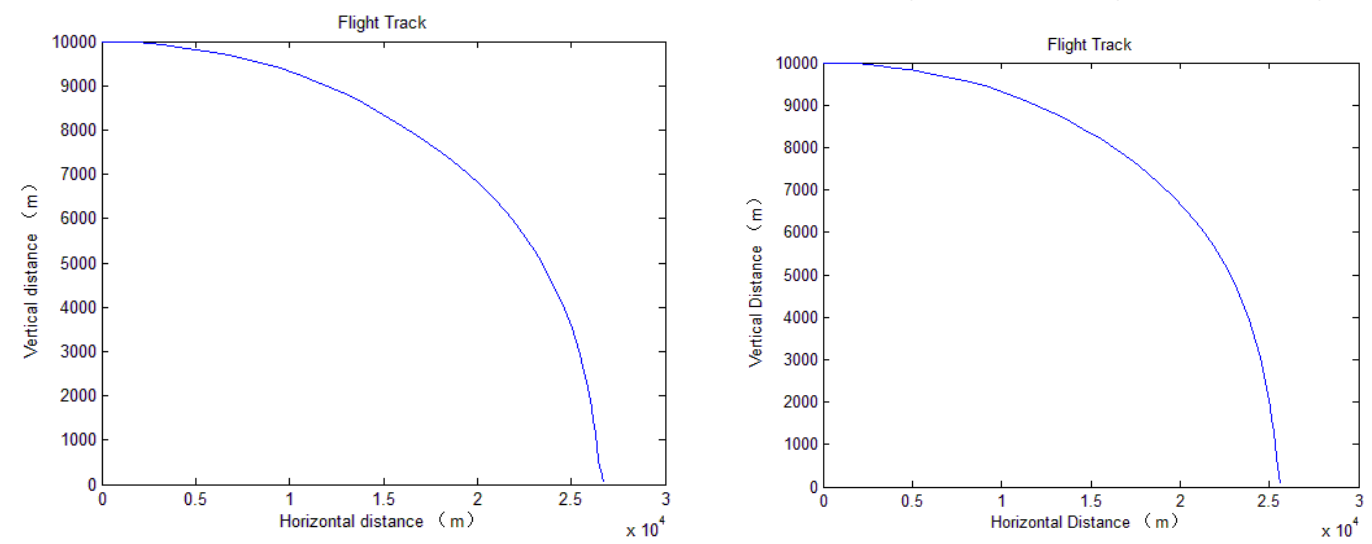

The figure shows: the horizontal displacement of Boeing 747-100 is 25589 meters, while that of Boeing 777-200 is 26687 meters. Taking into account that the weight difference is not obvious and the two types belong to the same tonnage models so the model can be considered a very good sensitivity.

\section{References}

[1]. Mingyuan Zhang, Hydro-mechanics,Beijing: HIGHER EDUCATION PRESS, 2010.

[2]. Jianghong Kuang, Bingliang Wang, Hongyan Lv,airplane mechanics, Beijing:Tsinghua University Press..

[3]. airplane knowledge and airplane science curriculum, http:///www.360doc.c, 2015-02-08. 